\title{
Analysis and Measurement of Forces in an Electrowetting-Driven Oscillator
}

Nathan Brad Crane ${ }^{1}$, Alex A Volinsky ${ }^{1}$, Vivek Ramadoss ${ }^{1}$, Michael Nellis ${ }^{1}$, Pradeep Mishra ${ }^{1}$, and Xiaolu Pang ${ }^{1,2}$

${ }^{1}$ Department of Mechanical Engineering, University of South Florida, 4202 E. Fowler Ave ENB 118, Tampa, FL, 33620

${ }^{2}$ Department of Materials Physics and Chemistry, University of Science and Technology Beijing, Beijing, 100083, China, People's Republic of

\begin{abstract}
Electrowetting is a promising method for manipulating small volumes of liquid on a solid surface. This complex phenomenon couples electrical and fluid properties and offers many potential surprises. The complex electrical and capillary interactions in electrowetting are illustrated by an analysis of an electrowetting configuration that produces an oscillating droplet motion from a steady DC voltage input. The paper presents an analysis of the electrowetting forces to explain the oscillation and presents a new method for measuring electrowetting forces using a Hysitron Triboindenter. Initial results are compared with predictions from numerical models and simplified analytical solutions.
\end{abstract}

\section{INTRODUCTION}

Electrowetting is the change of apparent surface energy in an applied electric field [1]. By varying the electric field applied to liquid drops, they can be moved, split, merged, and mixed to enable lab on a chip microfluidics [2]. Many applications of electrowetting have been proposed in recent years, and many different configurations have been studied. Cooney et.al. have considered the relative advantages of three different electrowetting configurations for control of droplet motion: two plate designs and variations of single plate designs in which all electrical connections are made from the bottom [3]. The electrowetting response is determined by interactions between the electrical and surface energies of the systems that can combine to produce a large variety of behaviors. A system in which oscillatory droplet motion is produced from a steady DC input along with an explanation for this behavior are presented. This phenomenon could be useful in improving mixing in digital microfluidic systems.

\section{$\underline{\text { Oscillation observations }}$}

This work considers the case in which both electrodes are located in the substrate and are isolated from the droplet by a dielectric layer. Test structures were fabricated by patterning aluminum electrodes on an oxidized silicon substrate and then spin coating an $800 \mathrm{~nm}$ layer of diluted Cytop 809M to act as both dielectric and hydrophobic layer. Uncovered $5 \mu$ droplets of $1 \mathrm{M} \mathrm{NaCl}$ water solution were positioned asymmetrically over the aluminum pads. When a DC potential is applied, liquid drops frequently exhibit an oscillatory response at around $35 \mathrm{~V}$. The 
motion frequency varied from 2 to $7 \mathrm{~Hz}$ with an oscillation amplitude of approximately 100-150 $\mu \mathrm{m}$. The oscillation was seen less often with thicker Cytop coatings. The center of the oscillation is offset from the electrode boundary.

This oscillation is caused by variations in the electrowetting force with the droplet position. Electrowetting is commonly characterized by the change in contact angle on a substrate as a function of applied voltage. In the basic model, the contact angles $(\theta)$ will depend on the voltage $(V)$ between the pad and the droplet and the liquid wetting angle $\left(\theta_{0}\right)$ without the applied voltage. These can then be related to the thickness $(\delta)$ and dielectric strength $\left(\varepsilon_{0}, \varepsilon_{\mathrm{R}}\right)$ of the Cytop layer by the Young Lippman equation [4]:

$$
\cos \theta_{1}=\cos \theta_{o}+\frac{\varepsilon_{o} \varepsilon_{r} V^{2}}{2 \gamma_{l v} \delta}
$$

The system energy is found by modeling the fluid using the contact angle data and the Young Lippman equation. The forces acting on the droplet are calculated by differentiating the energy with respect to the displacement of interest. A closed form solution is developed for the electrowetting force on a drop confined between an electrically insulating cover positioned over two substrate electrodes as illustrated in Figure 1(a). These predictions are compared with numerical modeling of surface forces using Surface Evolver [5, 6] and experimental measurements performed with a nanoindenter.

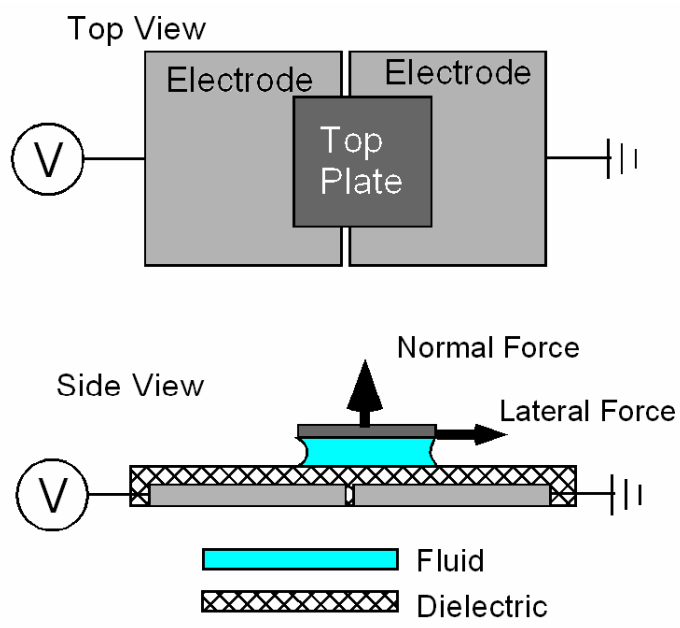

a)

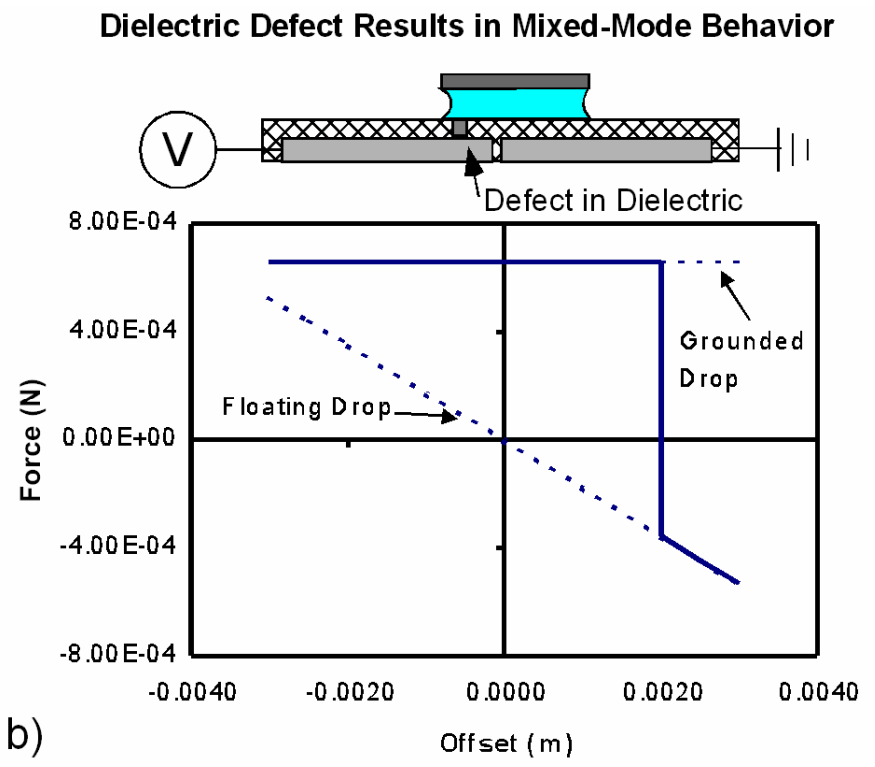

Figure 1. a) Schematic of the floating drop electrowetting configuration with square plate over the drop. b) Force displacement behavior of a mixed-mode drop with a transition at $0.002 \mathrm{~m}$ due to a defect in the dielectric layer. Dashed lines indicate the response of ideal grounded and floating droplets.

\section{$\underline{\text { Analytical droplet model }}$}

In the case of interest, the droplet is positioned over two electrodes with a voltage applied across the electrodes. The electrodes are covered with a dielectric material. The droplet can be considered conductive so that the system is modeled as two capacitors in series. The voltage between each pad and the droplet will vary with the droplet position due to changes in the 
capacitor area and thus their capacitance. Neglecting the droplet resistance, the arrangement can be modeled as simple series capacitor circuit composed of two parallel plate capacitors. The voltages across the left and the right capacitors $\left(V_{L}, V_{R}\right)$ are given as

$$
V_{L}=\frac{A_{R}}{A_{L}+A_{R}} V_{t o t}, V_{R}=\frac{A_{L}}{A_{L}+A_{R}} V_{t o t}
$$

The change in contact angle is driven by the reduction in effective surface energy by the energy stored in the capacitors. Using these relationships, the total capacitive energy is given by

$$
E=\frac{1}{2}\left(C_{l} V_{L}^{2}+C_{R} V_{R}^{2}\right)
$$

The area of the droplet over each electrode $\left(A_{L}, A_{R}\right)$ is a function of the droplet position and the form of this function depends on the droplet shape. In general, this shape will vary with the offset from the equilibrium position. However, if the droplet is sandwiched between the substrate and an electrically insulating cover plate that is wet by the liquid, the droplet contact area and its contact shape with the substrate will approach the area of the cover plate as the droplet thickness decreases relative to the cover plate dimensions. With this simplification, the area values of the droplet beneath a square cover plate with edge length $s$ can be related to the displacement $(x)$ of the cover plate from the equilibrium position:

$$
A_{L}=s\left(\frac{s}{2}-x\right), A_{R}=s\left(\frac{s}{2}+x\right),-\frac{s}{2}<x<\frac{s}{2}
$$

The energy and force as a function of position are then

$$
\begin{aligned}
& E=\frac{V_{t o t}^{2} \varepsilon_{0} \varepsilon_{R}}{8 \delta}\left(s^{2}-4 x^{2}\right) \\
& F_{x}=\frac{d E}{d x}=-\frac{\varepsilon_{0} \varepsilon_{R}}{\delta} V_{t o t}^{2} x
\end{aligned}
$$

where the force as a function of cover plate position is found by differentiating the energy with respect to the displacement. The static equilibrium position is at the center of the two electrodes with equal area on each electrode. In cases of limited viscous and electrical energy loss, the drop could oscillate around the equilibrium when disturbed. However, the oscillation would be centered on the gap and should not depend on the thickness of the dielectric coating. In contrast, the center of oscillations was offset from the electrode gap. While an offset could be caused by asymmetric electrowetting [7], contact angle measurements on the Cytop substrate show modest polarity dependence at low voltages.

This behavior could be explained by the presence of a hole in the dielectric layer. A hole would short the capacitor on one side so that no voltage will be applied across the region of the droplet over the shorted electrode. The full voltage drop will occur across the other side. In this case, the electrowetting force will be constant with displacement. The force is given by

$$
F_{x}=-\frac{\varepsilon_{0} \varepsilon_{R}}{2 \delta} s V_{t o t}^{2}
$$

If there is a local defect over one of the electrodes, hybrid behavior could be observed in which there is a transition from one mode to another as illustrated in Figure 1b). Oscillation is likely in this situation because there is no point at which the force is zero as required for equilibrium. 


\section{Electrowetting force measurement}

Typically, electrowetting is characterized by the change in contact angle. If information is desired about the resulting energy or forces, this is estimated by modeling. This work proposes for the first time to directly measure these effects. This is particularly useful in characterizing complicated phenomena and detecting local phenomena that might be difficult to detect on the basis of a contact angle change.

Electrowetting forces were measured using a custom tip in a Hysitron Triboindenter. The probe was assembled by aligning a $9 \mathrm{x} 9 \mathrm{~mm}^{2}$ glass cover slip with the axes of the Hysitron Triboindenter optical system. A blunt tip is then placed into the Hysitron transducer and this is brought into contact with the center of the coverslip. A drop of cyanoacrylate adhesive is applied to bond the coverslip to the blunt tip and the assembly was allowed to cure overnight at room temperature. Forces are measured by applying a droplet of known volume over the electrodes and then positioning the indenter over the droplet. The indenter is brought down towards the substrate until it is completely wet by the liquid. Typical heights were $400 \mu \mathrm{m}$ for a $42 \mu \mathrm{l}$ drop and $250 \mu \mathrm{m}$ for a $30 \mu \mathrm{l}$ drop. After each test, the droplet was replaced with a new droplet to reduce the impact of evaporation and contact angle hysteresis on the test results.

The test configuration was modeled using Surface Evolver to verify that the area of the liquid contact was adequately approximated by the assumption of a square profile. This was accomplished by relating the surface energy of the faces to the actual area over each electrode as calculated in the Surface Evolver model. After adjusting the analytical model for the effects of a finite gap between the electrodes, the areas agreed to within $1.5 \%$.

\section{RESULTS AND DISCUSSION}

Initially data was collected at zero voltage to test the stability of the measurement system. Figure 2a) shows a typical plot of the lateral and normal forces. A significant drift in the normal force is observed due to the liquid evaporation. However, the lateral force is relatively stable. The average drift rates measured for the normal and lateral forces were -0.835 and $-0.085 \mu \mathrm{N} / \mathrm{s}$, respectively. This work focuses on the measurement of the lateral forces.
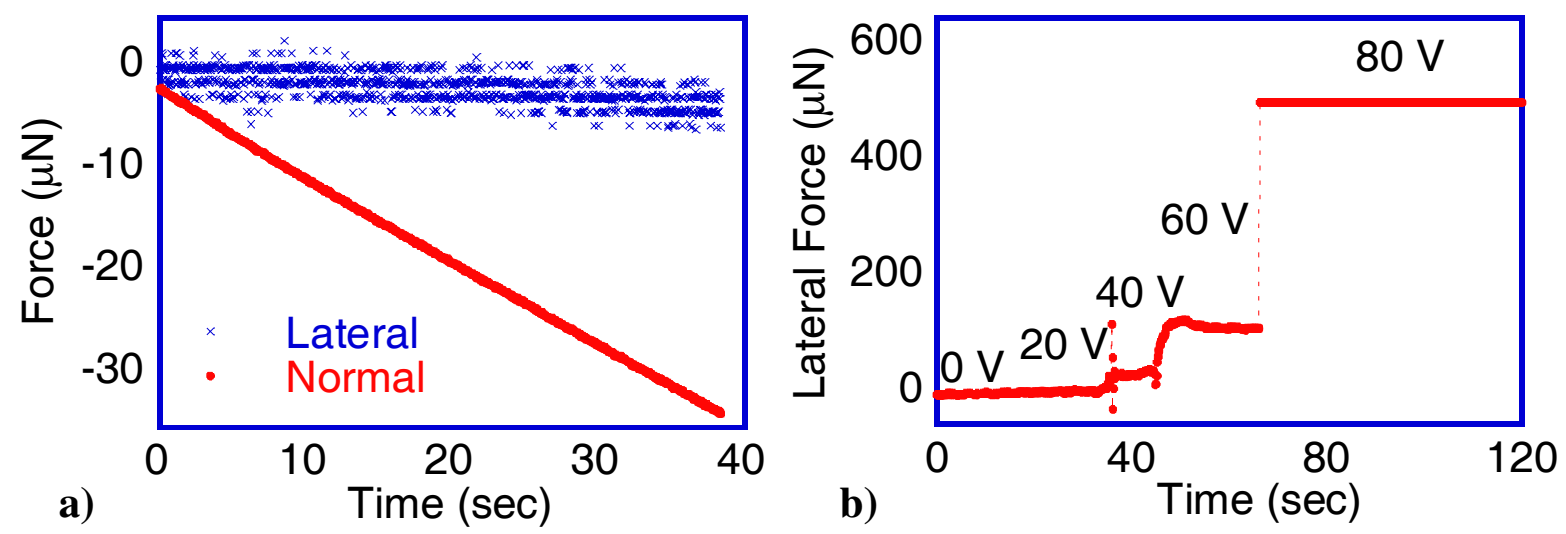

Figure 2. Electrowetting force data. a) Normal and lateral force measurements with zero applied voltage. b) Lateral force measured with steps of 0, 20, 40, 60, and $80 \mathrm{~V}$ applied. 
Figure $2 \mathrm{~b}$ ) shows the data from a $0.1 \mathrm{M} \mathrm{NaCl}$ drop with voltage steps of $0,20,40,60,80$ $\mathrm{V}$. The last three were clearly measured. These values were compared to theoretical predictions and good agreement was observed at 40 and $60 \mathrm{~V}$ when modeled as a floating drop. The YoungLippman equation was fit to the contact angle measurements for $1 \mathrm{M} \mathrm{NaCl}$ solution for a voltage range of $0-50 \mathrm{~V}$ to find an effective thickness of $1 \mu \mathrm{m}$ when assuming a relative dielectric constant of 2.1. The $80 \mathrm{~V}$ measurement is matched well by the grounded droplet model. This suggests that the coating on one side failed at the higher voltage. These results are summarized in Table I.

Table I. Comparison of measured electrowetting forces with analytical predictions.

\begin{tabular}{|c|c|c|c|}
\hline $\begin{array}{c}\text { Applied Voltage } \\
(\mathbf{V})\end{array}$ & $\begin{array}{c}\text { Measured Force } \\
(\mu \mathbf{N})\end{array}$ & $\begin{array}{c}\text { Predicted Force } \\
(\boldsymbol{\mu N})\end{array}$ & Prediction Method \\
\hline 20 & 6 & 11 & Floating Drop \\
\hline 40 & 41 & 44 & Floating Drop \\
\hline 60 & 113 & 98 & Floating Drop \\
\hline 80 & 505 & 535 & Grounded Drop \\
\hline
\end{tabular}

A series of droplets were also measured with a constant $100 \mathrm{~V}$ applied but with different offsets from the electrode gap. These tests were conducted with DI water to reduce potential degradation of the coating. A typical dataset from these tests is illustrated in Figure 3a). From this data the peak and steady forces were extracted and summarized in Figure $3 b$ ) as a function of the central position offset. This data shows a possible transition from floating to grounded drops as indicated above.

These early results suggest that this system can be effective in measuring electrowetting forces and can provide insight into experimental observations of droplet behavior. These early measurements have detected force transitions indicative of coating defects. With further refinement, these methods could be used to provide quick characterization of electrowetting materials and characterize the ability of electrowetting drops to apply forces to other interacting elements. This method can also provide a means to directly measure other parameters of interest in electrowetting such as dielectric decay and the influence of liquid conductivity on system response.
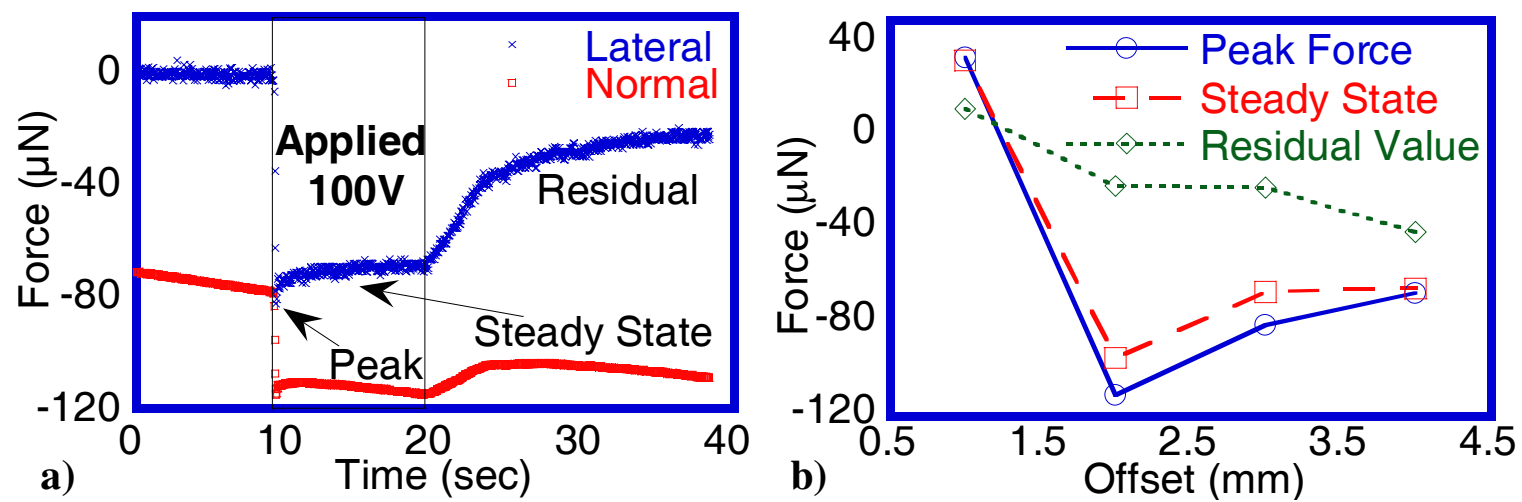

Figure 3. Electrowetting force measurements. a) Representative data sample with $100 \mathrm{~V}$ applied. b) Force measurements variation with position for DI water drops. Sign reversal is consistent with local shorting or dielectric breakdown. 


\section{CONCLUSIONS}

When electrowetting is done with a series capacitance circuit, the resulting force is shown to depend on the area of contact with the electrodes. The voltage across each capacitor is a function of the area of the electrode covered by the liquid drop. This can be used to create an oscillating drop motion from a steady voltage input when there is an abrupt transition between the circuit characteristics.

Understanding of the force variations will enable improvements in electrowetting droplet control and simplified droplet control schemes that reduce the number of independent electrodes required for simple droplet motions. The novel method for electrowetting force measurement presented here will also enable better understanding of anomalous behavior observed in many electrowetting systems.

\section{ACKNOWLEDGEMENTS}

Nathan Crane would like to acknowledge the support of the University of South Florida Research Education Initiative Program under Grant Number FMMD04. Alex Volinsky would like to acknowledge the support from NSF under CMMI-0631526 and CMMI-0600231 grants.

\section{REFERENCES}

[1] F. Mugele, J. C. Baret and D. Steinhauser, "Microfluidic mixing through electrowettinginduced droplet oscillations," Appl. Phys. Lett., vol. 88, pp. 204106, MAY 15. 2006.

[2] S. K. Cho, H. J. Moon and C. J. Kim, "Creating, transporting, cutting, and merging liquid droplets by electrowetting-based actuation for digital microfluidic circuits," J Microelectromech Syst, vol. 12, pp. 70-80, FEB. 2003.

[3] C. G. Cooney, C. Y. Chen, M. R. Emerling, A. Nadim and J. D. Sterling, "Electrowetting droplet microfluidics on a single planar surface," Microfluid. Nanofluid., vol. 2, pp. 435-446, SEP. 2006.

[4] F. Mugele and J. C. Baret, "Electrowetting: From basics to applications," J. Phys. -Condes. Matter, vol. 17, pp. R705-R774, JUL 20. 2005.

[5] K. Brakke, "The Surface Evolver," Exp. Math, vol. 1, pp. 141, 1992.

[6] J. Lienemann, A. Greiner and J. G. Korvink, "Modeling, Simulation, and optimization of electrowetting," IEEE Trans. Comput. -Aided Des. Integr. Circuits Syst. (USA), vol. 25, pp. 234, 2006/02/.

[7] S. K. Fan, H. P. Yang, T. T. Wang and W. Hsu, "Asymmetric electrowetting - moving droplets by a square wave," Lab on a Chip, vol. 7, pp. 1330-1335, 2007. 